\title{
Periodic Solutions in a Mathematical Model for the Treatment of Chronic Myelogenous Leukemia
}

\author{
A. Halanay* \\ Department of Mathematics I, Politehnica University of Bucharest \\ 060042 Bucharest, Romania
}

\begin{abstract}
Existence and stability of periodic solutions are studied for a system of delay differential equations with two delays, with periodic coefficients. It models the evolution of hematopoietic stem cells and mature neutrophil cells in chronic myelogenous leukemia under a periodic treatment that acts only on mature cells. Existence of a guiding function leads to the proof of the existence of a strictly positive periodic solution by a theorem of Krasnoselskii. The stability of this solution is analysed.
\end{abstract}

Key words: periodic solution, guiding function, index of an isolated solution, stability, chronic myelogenous leukemia

AMS subject classification: 34C25, 34A26, 34K20, 92D25

\section{Introduction}

Chronic myelogenous leukemia (CML) is a disease of the blood that affects white blood cells. The distinct mark of this disease is a chromosomal abnormality (the Philadelphia chromosome, $\mathrm{Ph}$ ) that is specific to leukemic white cells and only to them. As a result of a translocation between a segment of chromosome 22 and a segment of chromosome 9 a new oncogene fusion protein, BCR-ABL, is formed (see [6], [20]). Treatment aims to eliminate the blood cells that contain the abnormal BCR-ABL fusion gene.

\footnotetext{
*E-mail: halanay@mathem.pub.ro
} 
The mathematical model that will be used to incorporate treatment's effects consists of two coupled delay differential equations. It relies on[4], [5] and [7] with one equation governing the stem cells' evolution and the other one, the neutrophils' compartment. Similar investigations, for stem cells' evolution can be found in [2], [3], [15], [16], [19].

Other types of models are analysed in [1], [17], [18]. Investigation of existence of periodic solutions induced by a periodic treatment at the stem cells level was carried on in [9], [10], [11].

Denote by $x_{1}$ the density of pluripotential non-proliferating stem cells (units $10^{6}$ cells $/ \mathrm{kg}$ ) and by $x_{2}$ the circulating leukocytes (units $10^{9}$ cells $/ \mathrm{kg}$ ). The stem cells can exit the non-proliferating compartment by re-entering the proliferating phase at a rate $\beta_{1}\left(x_{1}\right)=\beta_{0} \frac{\theta_{1}^{2 m}}{\theta_{1}^{2 m}+x_{1}^{2 m}}, m>1$ or by differentiating into the three cell lines (leukocytes, erythrocytes and platelets). We retain only the differentiation rate into leukocyte line, $\beta_{2}\left(x_{2}\right)=k_{0} \frac{\theta_{2}^{2 n}}{\theta_{2}^{2 n}+x_{2}^{2 n}}, n>1$. A strictly positive $\delta_{1}$ will account for the loss of stem cells due to differentiation into the other two cell lines.

When re-entered into the proliferative phase, stem cells divide during a time $\tau_{1}$ (days). Due to mortality during the proliferating phase, the number that enter the non-proliferating phase will be

$$
2 e^{-\gamma_{1} \tau_{1}} \beta_{1}\left[x_{1}\left(t-\tau_{1}\right)\right] x_{1}\left(t-\tau_{1}\right) .
$$

The evolution of the leukocyte cells is influenced by a constant mortality rate $\delta_{2}>0$ and an amplification factor $A_{N}$. To these, treatment's effects are present through a function $u(t) \geq 0$ that diminishes the number of (leukemic) white blood cells. The number of leukocytes is increased by the number of pluripotent stem cells that differentiated during a time $\tau_{2}$, at a rate $\beta_{2}\left[x_{2}\left(t-\tau_{2}\right)\right]$, amplified by the factor $A_{N}$.

The equations of the model are

$$
\begin{aligned}
\dot{x}_{1} & =-\delta_{1} x_{1}-\beta_{0} \frac{\theta_{1}^{2 m}}{\theta_{1}^{2 m}+x_{1}^{2 m}} x_{1}-k_{0} \frac{\theta_{2}^{2 n}}{\theta_{2}^{2 n}+x_{2}^{2 n}} x_{1}+ \\
& +2 e^{-\gamma_{1} \tau_{1}} \beta_{0} \frac{\theta_{1}^{2 m} x_{1}\left(t-\tau_{1}\right)}{\theta_{1}^{2 m}+x_{1}\left(t-\tau_{1}\right)^{2 m}} \\
\dot{x}_{2} & =-\delta_{2} x_{2}-u(t) x_{2}+A_{N} k_{0} \frac{\theta_{2}^{2 n} x_{1}\left(t-\tau_{2}\right)}{\theta_{2}^{2 n}+x_{2}\left(t-\tau_{2}\right)^{2 n}}
\end{aligned}
$$

Introduce new variables through $\tilde{x}_{1}=\frac{1}{\theta_{1}} x_{1}, \tilde{x}_{2}=\frac{1}{\theta_{2}} x_{2}$. The system (1.1) becomes

$$
\begin{aligned}
\dot{\tilde{x}}_{1} & =-\delta_{1} \tilde{x}_{1}-\beta_{0} \frac{1}{1+\tilde{x}_{1}^{2 m}} \tilde{x}_{1}-k_{0} \frac{1}{1+\tilde{x}_{2}^{2 n}} \tilde{x}_{1}+ \\
& +2 e^{-\gamma_{1} \tau_{1}} \beta_{0} \frac{\tilde{x}_{1}\left(t-\tau_{1}\right)}{1+\tilde{x}_{1}\left(t-\tau_{1}\right)^{2 m}} \\
\dot{\tilde{x}}_{2} & =-\delta_{2} \tilde{x}_{2}-u(t) \tilde{x}_{2}+\frac{A_{N} \theta_{1}}{\theta_{2}} k_{0} \frac{\tilde{x}_{1}\left(t-\tau_{2}\right)}{1+\tilde{x}_{2}\left(t-\tau_{2}\right)^{2 n}}
\end{aligned}
$$


We denote by $\tilde{A}_{N}=A_{N} \frac{\theta_{1}}{\theta_{2}}$ the new amplification factor and return to the notations $x_{1}$ for the normalized stem cells density and $x_{2}$ for the normalized leukocyte density. Then, the model to be investigated is:

$$
\begin{aligned}
\dot{x}_{1} & =-\delta_{1} x_{1}-\beta_{0} \frac{x_{1}}{1+x_{1}^{2 m}}-k_{0} \frac{x_{1}}{1+\tilde{x}_{2}^{2 n}}+ \\
& +2 e^{-\gamma_{1} \tau_{1}} \beta_{0} \frac{x_{1}\left(t-\tau_{1}\right)}{1+x_{1}\left(t-\tau_{1}\right)^{2 m}} \\
\dot{x}_{2} & =-\delta_{2} x_{2}-u(t) x_{2}+\tilde{A}_{N} k_{0} \frac{x_{1}\left(t-\tau_{2}\right)}{1+x_{2}\left(t-\tau_{2}\right)^{2 n}} .
\end{aligned}
$$

Here $\delta_{1}, \delta_{2}, \beta_{0}, k_{0}, \tilde{A}_{N}$ are strictly positive constants, $m>1$ and $n>1$ (some exact values are to be found in [7]). The consideration of powers $2 \mathrm{~m}$ and $2 \mathrm{~m}$ in the Hill functions will allow for some global estimates that will play an important part in the proof of the existence of periodic solutions. We suppose that $\tau_{1}=k_{1} \tau$ and $\tau_{2}=k_{2} \tau$ with $k_{1}, k_{2}$ strictly positive integers and $\tau>0$. This is indeed the case for the data in [7] and also in all models where the delays are rational numbers.

We suppose $u$ is periodic with period $\tau$.

In section 2 it will be proved that the system (1.2) has a strictly positive $\tau$-periodic solution. The proof will employ the topological methods developed in [14]. In section 3 this $\tau$-periodic solution will be shown to be locally asymptotically stable. A section devoted to final comments on the results will conclude the paper.

\section{Existence of a strictly positive $\tau$-periodic solution of (1.2)}

Since $\tau_{1}=k_{1} \tau$ and $\tau_{2}=k_{1} \tau$, a $\tau$-periodic solution of (1.2) will be a solution of the ODE system with $\tau$-periodic coefficients

$$
\begin{aligned}
& \dot{x}_{1}=-\delta_{1} x_{1}-k_{0} \frac{x_{1}}{1+x_{2}^{2 n}}+\left(2 e^{-\gamma_{1} \tau_{1}}-1\right) \beta_{0} \frac{x_{1}}{1+x_{1}^{2 m}} \\
& \dot{x}_{2}=-\delta_{2} x_{2}-u(t) x_{2}+\tilde{A}_{N} k_{0} \frac{x_{1}}{1+x_{2}^{2 n}} .
\end{aligned}
$$

System (2.1) will be denoted as

$$
\dot{x}=f(t, x)
$$

so $f(t+\tau, x)=f(t, x), \quad \forall t \geq 0, \forall x=\left(x_{1}, x_{2}\right) \in \mathbf{R}^{2}$. Denote, for convenience,

$$
c_{0}=2 e^{-\gamma_{1} \tau_{1}}-1
$$

and suppose $c_{0}>0$ (data in [7] verify this).

Lemma 2.1. The function $\Phi: \mathbf{R}^{2} \rightarrow \mathbf{R}, \Phi\left(x_{1}, x_{2}\right)=-x_{1}^{2}-x_{2}^{2}$ is a guiding function for the system (2.1). 
Proof. By [14], Ch. 2, $\S 6.3$, we have to prove that $\exists R_{0}>0$ such that, for every $t \in[0, \tau]$ and every $x \in \mathbf{R}^{2}$ with $\|x\| \geq R_{0}$, if $f$ is given in (2.2),

$$
L_{f} \Phi=((\operatorname{grad} \Phi)(x), f(t, x))>0
$$

where $\left(\right.$, ) denotes the scalar product in $\mathbf{R}^{2}$ and $\|x\|$ is the euclidean norm. But

$$
\begin{aligned}
L_{f} \Phi= & 2 x_{1}^{2} \delta_{1}+2 k_{0} \frac{x_{1}^{2}}{1+x_{2}^{2 n}}-2 c_{0} \beta_{0} \frac{x_{1}^{2}}{1+x_{1}^{2 m}}+ \\
& +2\left[u(t)+\delta_{2}\right] x_{2}^{2}-2 \tilde{A}_{N} k_{0} \frac{x_{1} x_{2}}{1+x_{2}^{2 n}} .
\end{aligned}
$$

Since $m>1$ it follows that there exists $M_{1} \geq 1$ such that $\frac{x_{1}^{2}}{1+x_{1}^{2 m}} \leq M_{1}$ for every $x_{1} \in \mathbf{R}$. Then $-2 c_{0} \beta_{0} \frac{x_{1}^{2}}{1+x_{1}^{2 m}}>-2 c_{0} \beta_{0} M_{1}, \forall x_{1} \in \mathbf{R}$. Since $n>1$ there exists $M_{2} \geq 1$ such that

$$
A_{N} k_{0} \frac{\left|x_{2}\right|}{1+x_{2}^{2 n}} \leq M_{2} \quad \forall x_{2} \in \mathbf{R}
$$

Then

It follows that

$$
-2 \tilde{A}_{N} k_{0} \frac{x_{1} x_{2}}{1+x_{2}^{2 n}}>-2 A_{N} k_{0} \frac{\left|x_{1} x_{2}\right|}{1+x_{2}^{2 n}}>-2 M_{2}\left|x_{1}\right|
$$

$$
\begin{gathered}
L_{f} \Phi>2 \delta_{1} x_{1}^{2}+2 k_{0} \frac{x_{1}^{2}}{1+x_{2}^{2 n}}+2\left[u(t)+\delta_{2}\right] x_{2}^{2}-2 M_{2}\left|x_{1}\right|- \\
-2 c_{0} \beta_{0} M_{1}>2\left|x_{1}\right|\left(\left|x_{1}\right| \delta_{1}-M_{2}\right)+2\left[u(t)+\delta_{2}\right] x_{2}^{2}-2 c_{0} \beta_{0} M_{1} \rightarrow \infty
\end{gathered}
$$

if $\|x\| \rightarrow \infty, \forall t \in[0, \tau]$, so (2.3) is verified for every $t \in[0, \tau]$ and $\|x\|$ large enough.

Remark. (2.4) holds also when $c_{0}<0$.

Recall now Theorem 7.3 from [14]: Let $x^{*}$ be a $\tau$-periodic solution of (2.2) and let $\Phi$ be a guiding function of (2.2) with nonzero index. If the index of $x^{*}$, denoted $\gamma\left(x^{*}\right)$, is different from the index of $\Phi$, denoted $\gamma(\Phi, \infty)$, then (2.2) has a $\tau$-periodic solution different of $x^{*}$.

In the case of (2.1), $x^{*}=0$. Following [14] denote by $U(\tau)$ the shift operator on the trajectories of the solutions of (2.1), $U(\tau) x=\varphi(\tau ; 0, x)$ is the solution of (2.1) that at the initial moment $t=0$ takes the values $\varphi(0)=x$, calculated in $\tau$. Define also

$$
Q x=x-U(\tau) x, \quad x \in \mathbf{R}^{2} .
$$

Obviously $Q 0=0$. By [14], Ch. 2, $\S 5, \gamma\left(x^{*}\right)=\gamma\left(Q, C_{r}[0]\right)$ if $r$ is small enough. Here $\gamma\left(Q, C_{r}[0]\right)$ is the rotation number of $Q$ on the circle $C_{r}[0]$. Theorem 5.1 in [14] asserts that if for some smooth closed curve $L=\partial G$ with $0 \in \stackrel{\circ}{G}, \gamma(Q, L) \neq \gamma\left(x^{*}\right)$ then system (2.1) has a nonzero periodic solution. 
Proposition 2.2. Suppose

$$
c_{0} \beta_{0}-\delta_{1}-k_{0}>0
$$

Then $\gamma\left(x^{*}\right)=-1$.

Proof. Apply Theorem 7.1 in [14], Ch.2, §7. Consider

$$
A(t)=\frac{\partial f}{\partial x}(t, 0)=\left(\begin{array}{cc}
-\delta_{1}-k_{0}+c_{0} \beta_{0} & 0 \\
\tilde{A}_{N} k_{0} & -\delta_{2}-u(t)
\end{array}\right) .
$$

It is easily seen that the monodromy matrix of the system $\dot{x}=A(t) x$ is given by

$$
V(\tau)=\left(\begin{array}{cc}
e^{\tau\left(c_{0} \beta_{0}-k_{0}-\delta_{1}\right)} & 0 \\
\tilde{A}_{N} k_{0} \int_{0}^{\tau} e^{-\left(c_{0} \beta_{0}-\delta_{1}-k_{0}\right) s+\int_{\tau}^{s}\left[\delta_{2}+u(\theta)\right] d \theta} d s & e^{-\int_{0}^{\tau}\left[\delta_{2}+u(s)\right] d s}
\end{array}\right) .
$$

From (2.5) it follows that $V(\tau)$ has a multiplier greater then 1 and the result follows.

Lemma 2.3. The guiding function of $(2.1), \Phi\left(x_{1}, x_{2}\right)=-x_{1}^{2}-x_{2}^{2}$ has the index

$$
\gamma(\Phi, \infty)=1
$$

Proof. $(\operatorname{grad} \Phi)\left(x_{1}, x_{2}\right)=-2 I_{2}\left(\begin{array}{l}x_{1} \\ x_{2}\end{array}\right)$ and the result follows from [14], Ch.2, $\S 6$ formula (6.21) or Lemma 6.6.

Theorem 2.4. Suppose (2.5) holds. Then the system (2.1) has a strictly positive $\tau$-periodic solution (strictly positive is understood with respect to the standard cone in $\mathbf{R}^{2}$ so $x_{1}>0, x_{2}>0$ ).

Proof. Since, by Lemma 6.1 and Theorem 6.3 in [14], $\S 6$,

$$
\gamma(Q ; L)=(-1)^{2} \gamma(\Phi, \infty)=1 \neq \gamma(0)=-1
$$

the existence of a nonzero $\tau$-periodic solution follows from Theorem 7.3 in [14], Ch.2, $\S 7$.

In order to prove that (2.1) has a strictly positive $\tau$-periodic solution, remark first that if $x^{*}=$ $\left(0, x_{2}^{*}\right)$ is a $\tau$-periodic solution of $(2.1)$ then

$$
x_{2}^{*}(t)=e^{-\int_{0}^{t}\left[u(s)+\delta_{2}\right] d s} x_{2}^{*}(0)
$$

must be $\tau$-periodic and this happens if either $x_{2}^{*}(0)=0$ or if $\int_{0}^{\tau} u(s) d s+\delta_{2} \tau=0$ that is clearly impossible since $\delta_{2}>0$ and $u(s) \geq 0 \forall s$. So $x_{2}^{*} \equiv 0$. In the same vein, $\left(x_{1}^{*}, 0\right)$ is a solution of (2.1) if and only if $x_{1}^{*} \equiv 0$. It follows that, for $x^{*}$, the nonzero $\tau$-periodic solution of $(2.1), x_{1}^{*}(t) \neq 0$ and $x_{2}^{*}(t) \neq 0, \forall t$ so $x_{1}^{*}(0)>0$ and $x_{2}^{*}(0)>0$ imply $x_{1}^{*}(t)>0$ and $x_{2}^{*}(t)>0, \forall t$.

Remark also that if $x^{*}=\left(x_{1}^{*}, x_{2}^{*}\right)$ is a solution of (2.1) then $-x^{*}=\left(-x_{1}^{*},-x_{2}^{*}\right)$ is also a solution of (2.1) so, if (2.1) has a $\tau$-periodic solution with $x_{1}^{*}(t)<0$ and $x_{2}^{*}(t)<0, \forall t$ it has also a $\tau$-periodic solution with $x_{1}^{*}(t)>0$ and $x_{2}^{*}(t)>0, \forall t$. 
Suppose now that $x^{*}$ is a $\tau$-periodic solution of (2.1) such that $x_{1}^{*}(t)<0$ and $x_{2}^{*}(t)>0$, $\forall t \in[0, \tau]$. Then

$$
\dot{x}_{2}^{*}(t)=-\delta_{2} x_{2}^{*}(t)-u(t) x_{2}^{*}(t)+\tilde{A}_{N} k_{0} \frac{x_{1}^{*}(t)}{1+x_{2}^{*}(t)^{2 n}}<0
$$

for every $t \in[0, \tau]$ and this contradicts the fact that it must exist $t_{0} \in(0, \tau)$ such that $\dot{x}_{2}^{*}\left(t_{0}\right)=0$. If $x_{1}^{*}(t)>0$ and $x_{2}^{*}(t)<0, \forall t \in[0, \tau]$ then $\dot{x}_{2}^{*}(t)>0, \forall t \in[0, \tau]$ and a contradiction appears again.

It is thus proved that, under condition (2.5), the system (2.1) has a strictly positive $\tau$-periodic solution.

\section{Stability of the nonzero periodic solution}

Let $x^{*}=\left(x_{1}^{*}, x_{2}^{*}\right)$ be a nonzero periodic solution of (2.1). In order to study its stability we use the Theorem of stability by the first approximation for delay differential equations ([12] Theorem 18.3 or [13], $\S 1$, Theorem 1.9). In order to shorten the algebraic expressions introduce

$$
r(x)=\beta_{0} \frac{x}{1+x^{2 m}}
$$

and denote

$$
y_{\tau}(t)=y(t-\tau) .
$$

If the solution $x^{*}$ is translated into zero by

$$
y=x-x^{*}
$$

then $y=\left(y_{1}, y_{2}\right)$ satisfies

$$
\begin{aligned}
& \dot{y}_{1}=-\delta_{1} y_{1}-k_{0} \frac{y_{1}+x_{1}^{*}}{1+\left(y_{2}+x_{2}^{*}\right)^{2 n}}-r\left(y_{1}+x_{1}^{*}\right)+2 e^{-\gamma_{1} \tau_{1}} r\left(y_{1 \tau_{1}}+x_{1 \tau_{1}}^{*}\right)-\delta_{1} x_{1}^{*} \\
& \dot{y}_{2}=-\delta_{2} y_{2}-u(t) y_{2}+\tilde{A}_{N} k_{0} \frac{y_{1 \tau_{2}}+x_{1 \tau_{2}}^{*}}{1+\left(y_{2 \tau_{2}}+x_{2 \tau_{2}}^{*}\right)^{2 n}}-\delta_{2} x_{2}^{*}-u(t) x_{2}^{*}
\end{aligned}
$$

so

$$
\dot{y}=A(t) y+B(t) y_{\tau_{1}}+C(t) y_{\tau_{2}}+G\left(t, y_{\tau_{1}}, y_{\tau_{2}}\right)
$$

where $G$ contains the higher order terms and

$$
A(t)=\left[a_{i j}(t)\right]=\left(\begin{array}{cc}
-\delta_{1}-r^{\prime}\left(x_{1}^{*}\right)-\frac{k_{0}}{1+x_{2}^{* 2 n}} & \frac{2 n k_{0} x_{2}^{* 2 n-1} x_{1}^{*}}{\left(1+x_{2}^{* 2 n}\right)^{2}} \\
0 & -\delta_{2}-u(t)
\end{array}\right)
$$




$$
\begin{gathered}
B(t)=\left[b_{i j}(t)\right]=\left(\begin{array}{cc}
2 e^{-\gamma_{1} \tau_{1}} r^{\prime}\left(x_{1 \tau_{1}}^{*}\right) & 0 \\
0 & 0
\end{array}\right) \\
C(t)=\left[c_{i j}(t)\right]=\left(\begin{array}{cc}
0 & 0 \\
\frac{\tilde{A}_{N} k_{0}}{1+x_{2 \tau_{2}}^{* 2 n}} & -\frac{2 \tilde{A}_{N} k_{0} n x_{2 \tau_{2}}^{* 2 n-1} x_{1 \tau_{2}}^{*}}{\left(1+x_{2 \tau_{2}}^{* 2 n}\right)^{2}}
\end{array}\right) .
\end{gathered}
$$

The linearized around zero system corresponding to (3.4) is

$$
\dot{y}=A(t) y+B(t) y_{\tau_{1}}+C(t) y_{\tau_{2}}
$$

with $A, B, C$ matrix valued $\tau$-periodic functions.

To investigate the stability of the zero solution of (3.8) the criterion in [8], Theorem 4.18 will be used: if, for every continuous $\tau$-periodic function $g=\left(g_{1}, g_{2}\right)$ and for every $\lambda \in \mathbf{C}$ with $\left|e^{\lambda \tau}\right| \geq 1$, the system

$$
\dot{y}=A(t) y+B(t) y_{\tau_{1}}+C(t) y_{\tau_{2}}+e^{\lambda t} g
$$

has a solution $y=e^{\lambda t} h$ with $h(t+\tau)=h(t) \forall t$, then the zero solution of (3.8) is uniformly asymptotically stable.

When $y=e^{\lambda t} h, h=\left(h_{1}, h_{2}\right)$, is introduced into (3.9), it results that $h$ must verify the following system

$$
\begin{aligned}
& h_{1}^{\prime}=\left[a_{11}(t)-\lambda+b_{11}(t) e^{\lambda \tau_{1}}\right] h_{1}+a_{12}(t) h_{2}+g_{1}(t) \\
& h_{2}^{\prime}=c_{21}(t) e^{\lambda \tau_{2}} h_{1}+\left[-\delta_{2}-u(t)-\lambda+c_{22}(t) e^{\lambda \tau_{2}}\right] h_{2}+g_{2}(t)
\end{aligned}
$$

where $a_{i j}(t), b_{i j}(t)$ and $c_{i j}(t)$ are given in (3.5), (3.6) and respectively (3.7).

By [8], Ch. 3, §3.1, Theorem 3.1, the system (3.10) has, for every $\tau$-periodic $g$ and every $\lambda \in \mathbf{C}$ with $R e \lambda \geq 0$, a $\tau$-periodic solution if and only if the corresponding homogeneous system has no nonzero $\tau$-periodic solution. The homogeneous system corresponding to (3.10) can be written as (see (3.5), (3.6) and (3.7))

where $M=\left(\begin{array}{cc}-\lambda-\delta_{1} & 0 \\ 0 & -\lambda-\delta_{2}\end{array}\right)$ is obviously Hurwitz and $N(t+\tau)=N(t) \forall t$.

Let $\varphi$ be a solution of (3.11). Then

$$
\varphi(t)=e^{M t} \varphi(0)+e^{M t} \int_{0}^{t} e^{-M s} N(s) \varphi(s) d s .
$$

Let $\alpha>0$ be such that

$$
\left\|e^{M t}\right\| \leq e^{-\alpha t}, \quad \forall t \geq 0 \text { and } \forall \lambda \in \mathbf{C} \text { with } \operatorname{Re} \lambda \geq 0
$$

Suppose that

$$
a:=\sup _{t \in[0, \tau]}\|N(t)\|<\alpha
$$


Define $\psi(t)=e^{-M t} \varphi(t)$. It follows from (3.12) that

$$
\begin{aligned}
\|\psi(t)\| & =\|\varphi(0)\|+\int_{0}^{t}\|N(s)\|\|\psi(s)\| d s \leq \\
& \leq\|\varphi(0)\|+\int_{0}^{t} a\|\psi(s)\| d s
\end{aligned}
$$

Gronwall inequality implies that

$$
\|\psi(s)\| \leq\|\psi(0)\| e^{a t}
$$

Since $\varphi(t)=e^{M t} \psi(t)$ it follows that

$$
\|\psi(t)\| \leq\left\|e^{M t}\right\|\|\psi(t)\| \leq e^{-\alpha t} e^{a t}\|\varphi(0)\|=e^{-(\alpha-a) t}\|\varphi(0)\|
$$

so, by (3.14), $\lim _{t \rightarrow \infty}\|\varphi(t)\|=0$. Taking $t=n \tau$ it follows that $\varphi(0)=\varphi(n \tau) \rightarrow 0$ as $n \rightarrow \infty$ so $\varphi(0)$ must be zero for every $\tau$-periodic solution of (3.11) and (3.11) has no nonzero $\tau$-periodic solution.

Then (3.10) has a $\tau$-periodic solution $h$ for every $\tau$-periodic $g$ and the zero solution of (3.8) results asymptotically stable. The following theorem has been proved

Theorem 3.1. If $\alpha$ is defined by (3.13) and the condition (3.14) is fulfilled,the nonzero periodic solution of (2.1) is locally asymptotically stable.

Remark. Since for a matrix $A$ one has the estimate $\left\|e^{A t}\right\| \leq e^{\mu(A) t} \forall t \geq 0$, with

$$
\mu(A)=\frac{1}{2} \max \left\{\lambda \in \sigma\left(A+A^{*}\right)\right\}
$$

one can take $\alpha=\min \left\{\delta_{1}, \delta_{2}\right\}$.

\section{Discussion}

The treatment of CML with drugs active only on the mature neutrophil cells line was modeled using the model of lekopoiesis introduced in [5] and studied in more detail in [4] and also in [7] where a complete model of hematopoiesis is given with evolution of differentiated cells taken into account. Since the treatment is supposed to act only on mature white blood cells, this is the only equation that was considered for mature cells evolution. The influence of the other cell lines on stem cells' evolution has been relegated to a positive parameter that accounts for diminishing the number of non-proliferative stem cells. The role of this parameter is nevertheless essential in the proof of the existence of a specific guiding function for the system of ODE that results when existence of $\tau$-periodic solution is investigated.

Another hypothesis concerned the rational dependence of the two delays involved in the stem cells and respectively neutrophils equations. As mentioned before this is not very restrictive since all published data declare rational delays. 
The only assumption that had to be made in order to ensure that the index of the zero solution is different from the index of the guiding function is the positivity of the number $\left(2 e^{-\gamma_{1} \tau_{1}}-1\right)-\delta_{1}-k_{0}$. This condition is verified by the data in [7].

The main result of the paper is the existence of a $\tau$-periodic solution when a $\tau$-periodic treatment is administered. This solution is locally asymptotically stable under suitable conditions. This phenomenon might be worth mentioning and correlated to observed oscillatory behavior of blood cells' evolution in CML.

\section{Acknowledgements}

This work was supported by CNCSIS Grant ID-PCE-2011-3-0198.

\section{References}

[1] L.H. Abbott, F. Michor. Mathematical models of targeted cancer therapy. British Journal of Cancer 95 (2006), 1136-1141.

[2] M. Adimy, F. Crauste, A. Halanay, M. Neamţu, D. Opriş. Stability of Limit Cycles in a Pluripotent Stem Cell Dynamics Model. Chaos, Solitons\&Fractals, 27(4) (2006), 1091-1107.

[3] M. Adimy, F. Crauste, S. Ruan. A mathematical study of the hematopoiesis process with application to chronic myelogenous leukemia. SIAM J. Appl. Math. 65(4) (2005), 13281352.

[4] M. Adimy, F. Crauste, S. Ruan. Periodic oscillations in leukopoiesis models with two delays. Journal of Theoretical Biology 242 (2006), 288-299.

[5] S. Bernard, J. Belair, M.C. Mackey. Oscillations in cyclical neutropenia: new evidence based on mathematical modelling. J. Theor. Biology 223 (2003), 283-298.

[6] B. Clarkson, A. Strife, D. Wisniewski, U. Lambek, C. Liu. Chronic myelogenous leukemia as a paradigm of early cancer and possible curative strategies. Leukemia 17 (2003), 12111262.

[7] C. Colijn, M.C. Mackey. A mathematical model of hematopoiesis I-Periodic chronic myelogenous leukemia. J. Theor. Biology 237 (2005), 117-132.

[8] Aristide Halanay, Differential Equations: stability, oscilations, time lags. Academic Press, 1966.

[9] A. Halanay. Periodic Solutions in Mathematical Models for Hematological Diseases under Treatment. IEEE Proceedings of the 8-th IFAC Workshop on Time-Delay Systems, Sept. 1-3, Sinaia, Romania, 2009. 
[10] A. Halanay. Stability analysis for a mathematical model of chemotherapy action in hematological diseases. Bull. Sci. Soc. Roumaine Sci. Math. 53 (101) (2010), no. 1, 3-10.

[11] A. Halanay. Treatment induced periodic solutions in some mathematical models of tumoral cell dynamics. Mathematical Reports !2(62) (2010), no. 4, in press.

[12] J. Hale. Theory of Functional Differential Equations. Springer, New York, 1977.

[13] V. Kolmanovskii, A. Myshkis. Applied Theory of Functional Differential Equations. Kluwer Academic Publishers, Dordrecht, 1992.

[14] M.A. Krasnoselskii. Shift operator on orbits of differential equations. Nauka, Moskow, 1966 (in Russian).

[15] M.C. Mackey, A unified hypothesis of the origin of aplastic anemia and periodic hematopoiesis, Blood 51 (1978), 941-956.

[16] M.C. Mackey, C. Ou, L. Pujo-Menjouet, J. Wu. Periodic oscillations of blood cell population in chronic myelogenous leukemia. SIAM J. Math. Anal. 38 (2006), 166-187.

[17] F. Michor, T. Hughes, Y. Iwasa, S. Branford, N.P. Shah, C. Sawyers, M. Novak. Dynamics of chronic myeloid leukemia. Nature 435 (2005), 1267-1270.

[18] H. Moore, N.K. Li. A mathematical model for chronic myelogenous leukemia (CML) and T-cell interaction. J. Theor. Biol. 227 (2004), 513-523.

[19] L. Pujo-Menjouet, C. Mackey. Contribution to the study of periodic chronic myelogenous leukemia. Comptes Rendus Biol, 327 (2004), 235-244.

[20] C. Sawyers. Chronic Myeloid Leukemia. N. Engl. J. Med. 340 (2000), 1330-1340. 